\title{
Biclique Graphs of Interval Containment Bigraphs
}

\author{
Edmilson P. Cruz, Marina Groshaus, André L. P. Guedes ${ }^{\circledR}$
}

\begin{abstract}
The biclique graph, defined and characterized by Groshaus and Szwarcfiter in 2010, is the intersection graph of the bicliques of a graph. In 2021 Groshaus and Guedes introduced the mutually included biclique graph and proved that, for any triangle-free graph, its biclique graph is the square graph of its mutually included biclique graph. In this work we prove that the biclique graphs of interval containment bigraphs are square graphs of permutation graphs, generalizing a previous result about interval bigraphs (a subclass of interval containment bigraphs). Also, we prove that the mutually included biclique graphs of interval bigraphs have no more forbidden induced subgraphs than the permutation graphs.
\end{abstract}

\section{Introduction}

A graph is a containment graph of intervals $(\mathcal{C G} \mathcal{I})$ if its vertices can be represented by a family of closed intervals on the real line such that two vertices are adjacent if, and only if, one of the corresponding intervals contains the other. Such family of intervals is called an interval containment

2020 AMS Subject Classification: 05C75.

Keywords and Phrases: Biclique graphs, Interval Containment Bigraphs, Biclique.

This research was supported by CAPES, CNPq (428941/2016-8) and CONICET. 
model. $\mathcal{C G I}$ is equivalent to the class of permutation graphs [1, 4] and to the intersection of comparability graphs and co-comparability graphs [3]. A graph is an interval containment bigraph $(\mathcal{I C B})$ if its vertices can be represented by a bipartite family of closed intervals on the real line with partition $(X, Y)$ such that two vertices are adjacent if, and only if, they are from different parts and the interval in $X$ contains the interval in $Y$. Such family is called a bipartite interval containment model [9]. A graph is an interval bigraph $(\mathcal{I B G})$ if its vertices can be represented by a bipartite family of closed intervals on the real line such that two vertices are adjacent if, and only if, their corresponding intervals intersect and belong to different parts. Such family of intervals is called a bipartite interval model [7]. A biclique of a graph is a subset of its vertex set that induces a maximal complete bipartite graph. The biclique graph of a graph $G$ is the intersection graph of its bicliques. We denote the biclique graph of $G$ as $\mathrm{KB}(G)$. Biclique graphs were defined by Groshaus and Szwarcfiter in 2010 and the complexity of recognizing them is still open [6].

Two bicliques $B_{1}, B_{2}$ of a graph $G$ are said to be mutually included if one of the parts of $B_{1}$ is a subset of one of the parts of $B_{2}$ and viceversa. The mutually included biclique graph of $G$, denoted as $\operatorname{KB}_{\mathrm{m}}(G)$, is the graph whose vertices represent the bicliques of $G$ and two vertices are adjacent if, and only if, their corresponding bicliques are mutually included [5]. In Figure 1 (left), we show a graph with 3 bicliques. One of them is mutually included with the other two, but the others are not mutually included with each other. On the right side, the graph above is $\mathrm{KB}(G)$ and the one below is $\mathrm{KB}_{\mathrm{m}}(G)$.

For the following definitions, consider a bipartite family of intervals $M$ with partition $(X, Y)$ and a graph $G$ from one of the definitions above that accepts $M$ as its family of intervals. Consider that the endpoints of the intervals of $M$ are all distinct. It is always possible to get such a family of intervals. We call a set $B \subseteq M$ a biclique of intervals if the corresponding vertices of $G$ belong to a biclique. If $M$ is a bipartite interval model, we call $B$ a biclique of intersection of intervals. If $M$ is a bipartite interval 

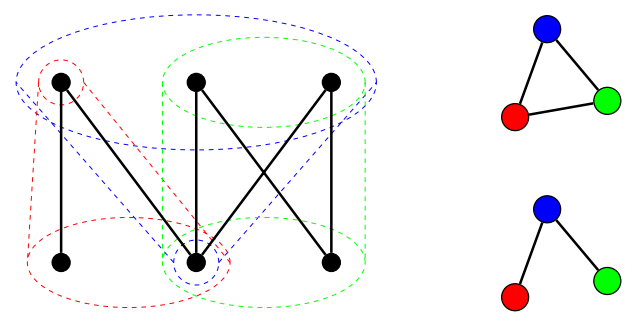

Figure 1: Graph $G$ with 3 bicliques, red, blue and green. On the right, the graph above is $\operatorname{KB}(G)$ and the one below is $\operatorname{KB}_{\mathrm{m}}(G)$.

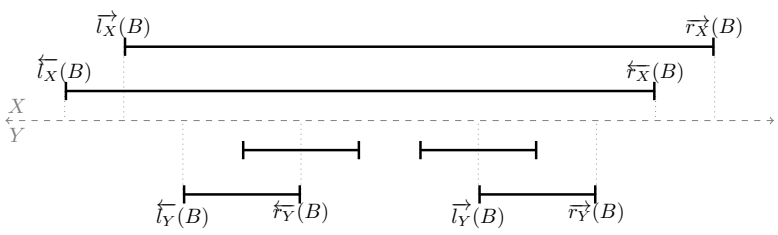

Figure 2: Biclique of intervals $B$ and the leftmost and rightmost endpoints of each part

containment model, then we call $B$ a biclique of containment of intervals instead.

For every interval $i \in M$, we denote the left endpoint of $i$ as $l(i)$ and the right endpoint of $i$ as $r(i)$. Consider the biclique of intervals $B$. We denote the leftmost left endpoint of $B \cap X$ as $\overleftarrow{l_{X}}(B)$ and the rightmost left endpoint of $B \cap X$ as $\overrightarrow{l_{X}}(B)$. Likewise, we denote leftmost right endpoint and rightmost right endpoint of $B \cap X$ respectively as $\overleftarrow{r_{X}}(B)$ and $\overrightarrow{r_{X}}(B)$. Similarly, for $B \cap Y$, we use $\overleftarrow{l_{Y}}(B), \overrightarrow{l_{Y}}(B), \overleftarrow{r_{Y}}(B)$, and $\overrightarrow{r_{Y}}(B)$. Figure 2 is an example of a bipartite family of intervals $M$ and a subset of intervals $B \subseteq M$. The endpoints from the definitions above, applied to $B$, are shown.

An asteroidal triple $(A T)$ is an independent set with 3 vertices such that for every pair of vertices there is a path connecting them while avoiding the neighbors of the third vertex [8]. See Figure 3 (left) for an example of 

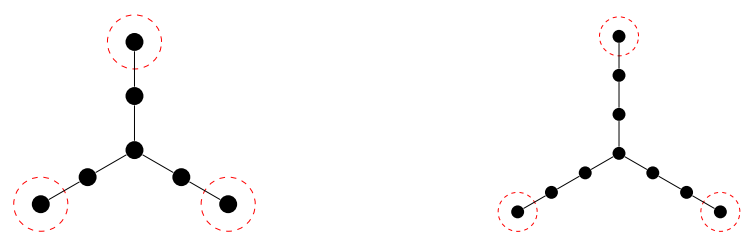

Figure 3: Example of an asteroidal-triple (left) and a bi-asteroidal triple (right). Note that the graph on the left is the same as the $\mathrm{KB}_{\mathrm{m}}$ graph of the graph on the right.

an $A T$. A bi-asteroidal triple (biAT) is an asteroidal triple such that the vertices of the path between each pair of vertices are not adjacent to the neighborhood of the third vertex [7]. See Figure 3 (right) for an example of a $b i A T$.

The square of a graph $G$ is the graph $G^{2}$ with the same vertex set as $G$ such that two vertices of $G^{2}$ are adjacent if and only if their distance in $G$ is at most 2 .

In this paper, we prove (in Section 2) that the biclique graphs of $\mathcal{I C B}$ are square of permutation graphs, generalizing our previous result on the biclique graphs of $\mathcal{I B G}[2]$. Note that $\mathcal{I B G} \subseteq \mathcal{I C B}$, as proven by Saha et al. 10. We also prove (in Section 3) that the mutually included biclique graphs of interval bigraphs have no more forbidden induced subgraphs than the permutation graphs. Furthermore, we present (in Section 44) some results that can help to increase our knowledge on the biclique graphs of bipartite graphs. For instance, for any bipartite graph $G$, we prove that if $\mathrm{KB}_{\mathrm{m}}(G)$ is $A T$-free then $G$ is $b i A T$-free, and that $G$ is $P_{4}$-free if and only if $E\left(\mathrm{~KB}_{\mathrm{m}}(G)\right)=\emptyset$.

\section{Square graph of permutation graphs}

In this section, we prove that every biclique graph of an interval containment bigraph is the square of a permutation graph. Consider the bipartite interval containment model $M$ with partition $(X, Y)$. For any 
pair of distinct bicliques of containment of intervals $B_{1}, B_{2} \subseteq M$, let $R$ and $R_{\text {co }}$ be the relations defined as

$$
\begin{aligned}
\left(B_{1}, B_{2}\right) \in R & \Longleftrightarrow\left(\overrightarrow{l_{X}}\left(B_{2}\right) \leq \overrightarrow{l_{X}}\left(B_{1}\right)\right) \wedge\left(\overleftarrow{r_{X}}\left(B_{1}\right) \leq \overleftarrow{r_{X}}\left(B_{2}\right)\right) \\
\left(B_{1}, B_{2}\right) \in R_{\mathrm{co}} & \Longleftrightarrow\left(\overrightarrow{l_{X}}\left(B_{1}\right)<\overrightarrow{l_{X}}\left(B_{2}\right)\right) \wedge\left(\overleftarrow{r_{X}}\left(B_{1}\right)<\overleftarrow{r_{X}}\left(B_{2}\right)\right)
\end{aligned}
$$

Lemma 2.1. For any pair of distinct bicliques of containment of intervals $B_{1}, B_{2} \subseteq M$, it holds that

$$
\left(B_{1}, B_{2}\right) \notin R \wedge\left(B_{2}, B_{1}\right) \notin R \Longleftrightarrow\left(B_{1}, B_{2}\right) \in R_{c o} \vee\left(B_{2}, B_{1}\right) \in R_{c o} .
$$

Proof. Consider $P\left(B_{1}, B_{2}\right) \Longleftrightarrow \overrightarrow{l_{X}}\left(B_{1}\right) \leq \overrightarrow{l_{X}}\left(B_{2}\right)$ and $Q\left(B_{1}, B_{2}\right) \Longleftrightarrow$ $\overleftarrow{r_{X}}\left(B_{1}\right) \leq \overleftarrow{r_{X}}\left(B_{2}\right)$. By the distribution law, we have that

$$
\begin{aligned}
& \left(B_{1}, B_{2}\right) \notin R \wedge\left(B_{2}, B_{1}\right) \notin R \\
\Longleftrightarrow & \left(\neg P\left(B_{2}, B_{1}\right) \vee \neg Q\left(B_{1}, B_{2}\right)\right) \wedge\left(\neg P\left(B_{1}, B_{2}\right) \vee \neg Q\left(B_{2}, B_{1}\right)\right) \\
\Longleftrightarrow & \left(\neg P\left(B_{2}, B_{1}\right) \wedge \neg P\left(B_{1}, B_{2}\right)\right) \vee\left(\neg P\left(B_{2}, B_{1}\right) \wedge \neg Q\left(B_{2}, B_{1}\right)\right) \\
& \vee\left(\neg Q\left(B_{1}, B_{2}\right) \wedge \neg P\left(B_{1}, B_{2}\right)\right) \vee\left(\neg Q\left(B_{1}, B_{2}\right) \wedge \neg Q\left(B_{2}, B_{1}\right)\right) .
\end{aligned}
$$

Cancelling the contradictions $\neg P\left(B_{2}, B_{1}\right) \wedge \neg P\left(B_{1}, B_{2}\right)$ and $\neg Q\left(B_{1}, B_{2}\right) \wedge$ $\neg Q\left(B_{2}, B_{1}\right)$, we have that

$$
\begin{array}{r}
\left(\neg P\left(B_{2}, B_{1}\right) \wedge \neg Q\left(B_{2}, B_{1}\right)\right) \vee\left(\neg Q\left(B_{1}, B_{2}\right) \wedge \neg P\left(B_{1}, B_{2}\right)\right) \\
\Longleftrightarrow\left(B_{1}, B_{2}\right) \in R_{\mathrm{co}} \vee\left(B_{2}, B_{1}\right) \in R_{\mathrm{co}}
\end{array}
$$

Groshaus and Guedes [5] proved that, for a $K_{3}$-free graph $G, \operatorname{KB}(G)=$ $\mathrm{KB}_{\mathrm{m}}(G)^{2}$ and given bicliques $B_{1}$ and $B_{2}$, with parts $X_{1}, Y_{1}$ and $X_{2}, Y_{2}$, respectively, if $X_{1} \subset X_{2}$ then $Y_{2} \subset Y_{1}$.

Theorem 2.1. The biclique graph of every interval containment bigraph is the square of a permutation graph. 


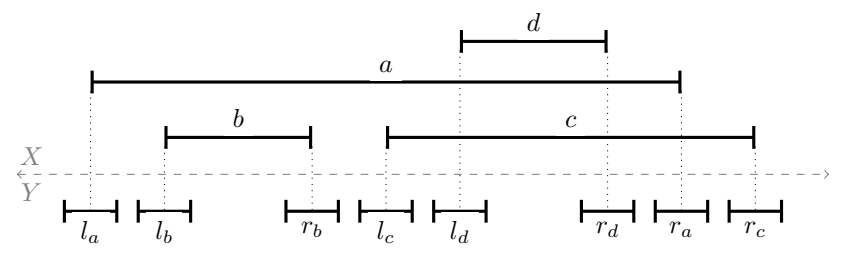

Figure 4: Bipartite interval model derived from an interval containment model

Proof. We can directly prove that the relations $R$ and $R_{\text {co }}$ are transitive and anti-symmetric, that is, they are strict partial orders. Let $G$ be an interval containment bigraph and $M$ be a bipartite interval model of $G$. Let $B_{1}, B_{2} \subseteq M$ be bicliques of containment of intervals. Suppose $B_{1}$ and $B_{2}$ are mutually included and w.l.o.g. that $\left(B_{2} \cap X\right) \subset\left(B_{1} \cap X\right)$. This implies that $\left(B_{1}, B_{2}\right) \in R$. Now, suppose $\left(B_{1}, B_{2}\right) \in R$, that is, $\left(\overrightarrow{l_{X}}\left(B_{2}\right) \leq \overrightarrow{l_{X}}\left(B_{1}\right)\right) \wedge\left(\overleftarrow{r_{X}}\left(B_{1}\right) \leq \overleftarrow{r_{X}}\left(B_{2}\right)\right)$. This implies that $\left(B_{2} \cap X\right) \subset$ $\left(B_{1} \cap X\right)$. As the graph is bipartite, this implies that $B_{1}$ and $B_{2}$ are mutually included [5, Lemma 3.1]. Consequently, $\operatorname{KB}_{\mathrm{m}}(G)$ is a comparability graph, with order $R$. By Lemma 2.1. $\mathrm{KB}_{\mathrm{m}}(G)$ is also a cocomparability graph with order $R_{\mathrm{co}}$. So, $\operatorname{KB}_{\mathrm{m}}(G)$ is a permutation graph as it is both a comparability graph and a co-comparability graph [3]. As $\mathrm{KB}(G)=\mathrm{KB}_{\mathrm{m}}(G)^{2}$, we have that $\mathrm{KB}(G)$ is the square of a permutation graph.

\section{Permutation graphs as induced subgraphs}

In this section, we prove that any permutation graph $G$ is an induced subgraph of a mutually included biclique graph of an interval bigraph by presenting a mapping between an arbitrary interval containment model $M$ and a subset of bicliques of an interval bigraph $H$ such that one interval of $M$ contains another if, and only if, their corresponding bicliques are mutually included. 
Theorem 3.1. If $G$ is a permutation graph, then $G$ is isomorphic to an induced subgraph of $\mathrm{KB}_{\mathrm{m}}(H)$ for some interval bigraph $H$.

Proof. Consider an interval containment model $M$. We define the partition $(X, Y)$ of the bipartite interval model $M^{\prime}$ as $X=M$ and $Y=\left\{l_{i}, r_{i} \mid\right.$ $i \in X\}$ such that $\{l(j), r(j) \mid j \in X\} \cap l_{i}=\{l(i)\}$ and $\{l(j), r(j) \mid j \in X\}$ $\cap r_{i}=\{r(i)\}$. We also define, for $i \in X$, the bicliques $B_{i}$ of $M^{\prime}$ as those that contain $\left\{i, l_{i}, r_{i}\right\}$ as a subset. For any distinct intervals $i, j \in B_{i} \cap X$, we have that $l(j)<l(i)$, since $j$ would not intersect $l_{i}$ otherwise. Thus, we have that $\overrightarrow{l_{X}}\left(B_{i}\right)=l(i)$. In the same way, we have that $\overleftarrow{r_{X}}\left(B_{i}\right)=r(i)$ Therefore, every interval in $Y$ that intersects $i$ also intersects every interval in $B_{i} \cap X$, since they contain $i$, and that $B_{i}$ is unique. We also have that $l_{j}$ does not intersect $i$, because $l(j)<l(i)$ and $l_{j}$ contains only $l(j)$ from the endpoints of intervals in $X$. Thus, we have that $B_{j} \neq B_{i}$. Therefore, there is a bijection between the intervals in $M$ and the bicliques $B_{i}$ of $M^{\prime}$. Because $\overrightarrow{l_{X}}\left(B_{i}\right)=l(i)$ and $\overleftarrow{r_{X}}\left(B_{i}\right)=r(i)$, we have for any distinct $i, j \in M$ that $l(j)<l(i) \wedge r(i)<r(j) \Longleftrightarrow \overrightarrow{l_{X}}\left(B_{j}\right)<\overrightarrow{l_{X}}\left(B_{i}\right) \wedge \overleftarrow{r_{X}}\left(B_{i}\right)<\overleftarrow{r_{X}}\left(B_{j}\right)$ Since every interval other than $j$ in $B_{j} \cap X$ contains $j$, they also intersect the intervals of $B_{i} \cap Y$. Thus, we have that $B_{j} \cap X \subset B_{i} \cap X$. If $j$ does not contain $i$, we have that $l(i)<l(j) \vee r(j)<r(i) \Longrightarrow \overrightarrow{l_{X}}\left(B_{i}\right)<$ $l(j) \vee r(j)<\overleftarrow{r_{X}}\left(B_{i}\right)$. Thus, we have that $l_{j} \notin B_{i}$ or $r_{j} \notin B_{i}$. Therefore, we have that $B_{j} \cap Y \not \subset B_{i} \cap Y$ and, since $j \notin B_{i}$, that $B_{i} \cap X \not \subset B_{j} \cap X$. By contrapositive, if $B_{i}$ and $B_{j}$ are mutually included, then $j$ contains $i$. Since $j$ contains $i$ if and only if $B_{i}$ and $B_{j}$ are mutually included, we have that the $\mathcal{C G}$ graph corresponding to $M$ is an induced subgraph of some $\mathrm{KB}_{\mathrm{m}}$ graph of an interval bigraph.

Figure 4 is an example of a bipartite interval model $M^{\prime}$ constructed using the definitions described in the proof above. As a consequence of Theorem 3.1, the mutually included biclique graphs of interval bigraphs have no more forbidden induced subgraphs than the permutation graphs. 


\section{Bipartite Graphs}

In this section we present some properties of $\mathrm{KB}_{\mathrm{m}}$ of bipartite graphs. These properties are proven by case analysis and the proofs are omitted. We say that two bicliques are edge-intersecting if they have a common edge. We say that they are vertex-intersecting if they are intersecting but are not edge-intersecting.

Theorem 4.1. The following statements hold for a bipartite graph $G$ : (i) if $\mathrm{KB}_{\mathrm{m}}(G)$ is AT-free then $G$ is biAT-free; (ii) $G$ is $P_{4}$-free if and only if $E\left(\mathrm{~KB}_{\mathrm{m}}(G)\right)=\emptyset$; (iii) if $G$ is $P_{5}$-free then there is no pair of vertexintersecting bicliques; (iv) $G$ is domino-free if and only if every pair of edge-intersecting bicliques are mutually included.

Proof ideas: (i) By construction, proving that if $G$ has a bi-asteroidal triple then $\mathrm{KB}_{\mathrm{m}}(G)$ has an asteroidal triple; (ii) By inspection of the possibilities of mutually included bicliques: if there is a pair of mutually included bicliques then there is a $P_{4}$ in $G$ (see Figure 5 (left)), if there is a $P_{4}$, there are at least two bicliques that are mutually included; (iii) By inspection of the possibilities of vertex-intersecting bicliques: if there is a pair of vertex-intersecting bicliques then there is a $P_{5}$ in $G$ (see Figure 5 (middle)); and (iv) By inspection of the possibilities of edge-intersecting bicliques that are not mutually included: if there is a pair of not mutually included edge-intersecting bicliques then there is a domino in $G$ (see Figure 5 (right)), if there is a domino, there are at least two bicliques that are edge-intersecting that are not mutually included. 

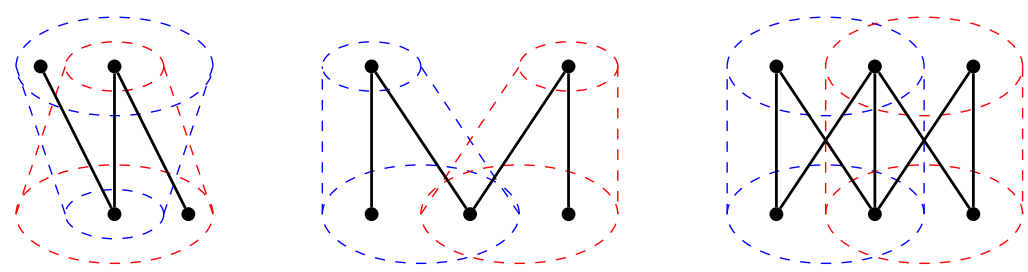

Figure 5: Edge types of bicliques that are: mutually included and a $P_{4}$ (left), vertex-intersecting and a $P_{5}$ (middle), and edge-intersecting not mutually included and a domino (right).

\section{References}

[1] A. Brandstädt, V. B. Le, and J. P. Spinrad. Graph Classes: a Survey. SIAM Monographs on Discrete Mathematics and Applications. Society for Industrial and Applied Mathematics (SIAM), Philadelphia, PA, 1999.

[2] E. P. Cruz, M. Groshaus, A. L. P. Guedes, and J. P. Puppo. Biclique graphs of interval bigraphs. Discrete Applied Mathematics, 281:134$143,2020$.

[3] Ben Dushnik and E. W. Miller. Partially ordered sets. American Journal of Mathematics, 63(3):600-610, 1941.

[4] Martin Charles Golumbic. Algorithmic graph theory and perfect graphs. Academic Press New York, 1980.

[5] Marina Groshaus and André L. P. Guedes. Biclique graphs of $K_{3}$-free graphs and bipartite graphs. In XI Latin and American Algorithms, Graphs and Optimization Symposium, 2021.

[6] Marina Groshaus and Jayme L. Szwarcfiter. Biclique graphs and biclique matrices. Journal of Graph Theory, 63(1):1-16, 2010. 
[7] Frank Harary, Jerald A. Kabell, and Frederick R. McMorris. Bipartite intersection graphs. Commentationes Mathematicae Universitatis Carolinae, 023(4):739-745, 1982.

[8] C. Lekkeikerker and J. Boland. Representation of a finite graph by a set of intervals on the real line. Fundamenta Mathematicae, 51(1):4564, 1962.

[9] Min Chih Lin and Jayme L. Szwarcfiter. Characterizations and recognition of circular-arc graphs and subclasses: A survey. Discrete Mathematics, 309(18):5618-5635, 2009. Combinatorics 2006, A Meeting in Celebration of Pavol Hell's 60th Birthday (May 1-5, 2006).

[10] Pranab K. Saha, Asim Basu, Malay K. Sen, and Douglas B. West. Permutation bigraphs and interval containments. Discrete Applied Mathematics, 175:71-78, 2014.

Edmilson Pereira da Cruz

Curitiba - PR, Brazil

Departamento de Informática

Universidade Federal do Paraná

epcruz@inf.ufpr.br
Marina Groshaus

Curitiba - PR, Brazil

Departamento Acadêmico de

Informática

Universidade Tecnológica

Federal do Paraná

Universidad de Buenos Aires

CONICET / Argentina

marinagroshaus@utfpr.edu.br

André Luiz Pires Guedes

Curitiba - PR, Brazil

Departamento de Informática

Universidade Federal do Paraná

andre@inf.ufpr.br 